Disclosure of Interest: None declared

DOI: 10.1136/annrheumdis-2018-eular.1186

\section{THU0225 THE PROGRESSION OF LUNG INVOLVEMENT IN RHEUMATOID ARTHRITIS PATIENTS RECEIVING BIOLOGICAL THERAPIES}

Y. Yalçınkaya ${ }^{1}$, M. Can ${ }^{1}$, S. Karakurt ${ }^{2}$, N. Coşkun ${ }^{3}$, E. Albayrak ${ }^{4}$, H. Mursaloglu ${ }^{4}$, A. Topcu ${ }^{5}$, M. Demir ${ }^{5}$, E. Dalkllic ${ }^{3}$, N. Inanc ${ }^{1} .{ }^{1}$ Department of Internal Medicine, Division of Rheumatology, ${ }^{2}$ Department of Pulmonary Medicine, Marmara University, School of Medicine, Istanbul; ${ }^{3}$ Department of Internal Medicine, Division of Rheumatology, Uludag University, Bursa; ${ }^{4}$ Marmara University, School of Medicine, Istanbul, Turkey; ${ }^{5}$ Department of Internal Medicine, Marmara University, School of Medicine, Istanbul, Turkey

Background: Lung involment is one of the extra-articular manifestations that is shown to be related to morbidity and mortality in Rheumatoid arthritis (RA).

Objectives: We evaluated the progression of lung involvement in RA patients receiving biological treatment.

Methods: Eighty RA patients fulfilling ACR/EULAR classification criteria (2010) receiving biologics who had high-resolution computerised tomography (HRCT) of chest within 6 months of respiratory symptoms (cough/dyspnea) and/or any pathology in chest radiography were included. Warrick score, evaluating alveolitis and fibrosis, was calculated in RA patients by using HRCT of chest.

Results: The demographics and clinical findings were summarised in table 1. In 29 RA patients with interstitial findings in HRCT of chest; 7 (24\%) were regressed and $1(4 \%)$ was progressed with biologic drugs. Findings of alveolitis and fibrosis were completely regressed in 2 , only alveolitis component was completely regressed in 3 and partially in 2 patients. The patient who progressed had new findings of alveolitis (table 2). Twenty-one patients in whom Warrick scores did not change, 5 received rituximab (RTX) and 10 received TNF inhibitors (TNF-inh). The mean Warrick scores was improved after biologics $(11,3 \pm 9(3-30)$ vs $10,3 \pm 9$ $(0-30), p=0,035)$. The improvement in alveolitis scores was significant $(2,1$ $\pm 1,6$ vs $1,5 \pm 1,6, p=0,031)$ while fibrosis scores were not $(9,2 \pm 7,7$ vs $8,8 \pm 8)$. Initial and control Warrick scores were higher in RTX receivers ( $16 \pm 9$ vs $9,2 \pm 8$, $\mathrm{p}=0034$ and $14,8 \pm 10,1$ vs $8,4 \pm 8,1$ ), but the improvement after biologics was not different.

Abstract THU0225 - Table 1. Demographics in Rheumatoid Arthritis Patients

\begin{tabular}{|l|l|}
\hline & $\mathrm{n}=80$ \\
\hline Age(year) & $53 \pm 12$ \\
\hline Gender(FM) & $61 / 19$ \\
\hline Disease duration(year) & $12 \pm 9$ \\
\hline Smoking(\%) & $26 \%$ \\
\hline RF(+) & $64(80 \%)$ \\
\hline AnticCP(+) & $50(63 \%)$ \\
\hline DAS28-ESR-after biologics & $3,3 \pm 1,1$ \\
\hline
\end{tabular}

\begin{tabular}{|c|c|c|c|c|c|c|c|}
\hline Scores & $\begin{array}{l}\text { Initial } \\
\text { Warrick- } \\
\text { Alveolitis }\end{array}$ & $\begin{array}{l}\text { Initial } \\
\text { Warrick- } \\
\text { Fibrosis }\end{array}$ & $\begin{array}{l}\text { Initial } \\
\text { Warrick- } \\
\text { Total }\end{array}$ & $\begin{array}{l}\text { Control } \\
\text { Warrick- } \\
\text { Alveolitis }\end{array}$ & $\begin{array}{l}\text { Control } \\
\text { Warrick- } \\
\text { Fibrosis }\end{array}$ & $\begin{array}{l}\text { Control } \\
\text { Warrick: } \\
\text { Total }\end{array}$ & Current Therapy \\
\hline Case 1 & 3 & 8 & 11 & 0 & 0 & 0 & RTX+MTX, seropositive \\
\hline Case 2 & 3 & 5 & 8 & 0 & 0 & 0 & TNFi+MTX, seropositive \\
\hline Case 3 & 3 & 8 & 11 & 2 & 8 & 10 & RTX, seropositive \\
\hline Case 4 & 4 & 17 & 21 & 3 & 17 & 20 & RTX, seropositive \\
\hline Case 5 & 3 & 0 & 3 & 0 & 0 & 0 & TNFi+MTX, seropositive \\
\hline Case 6 & 3 & 0 & 3 & 0 & 0 & 0 & TNFi+MTX,seronegative \\
\hline Case 7 & 3 & 4 & 7 & 0 & 4 & 4 & TNFi+MTX, seronegative \\
\hline Case 8 & 0 & 3 & 3 & 2 & 3 & 5 & RTX+MTX, seropositive \\
\hline
\end{tabular}

Abstract THU0225 - Table 2. Progression of Warrick Scores in Cases After Biologics

Conclusions: This study revealed an improvement in alveolitis after biologic drugs in RA patients. Fibrosis was not found to be effected by therapy. Lung lesions were improved in both RTX and TNF-inh groups.

Disclosure of Interest: None declared

DOI: 10.1136/annrheumdis-2018-eular.5823
THU0226

SERIAL INTERFERON-GAMMA RELEASE ASSAYS IN PATIENTS WITH RHEUMATOID ARTHRITIS TREATED WITH BIOLOGIC AGENTS

Y.J. Huang, Y.F. Fang, Y.F. Chen, P.H. Tsai, C.F. Kuo, K.H. Yu, S.F. Luo. Division of Rheumatology, Allergy and Immunology, Chang Gung Memorial Hospital and Chang Gung University, Taoyuan, Taiwan, Province of China

Background: It is increasing attention about the risk of latent tuberculosis infection (LTBI) reactivation during the treatment of biologic agents for patients with rheumatoid arthritis. Taiwan rheumatology association recommend performing at least annual interferon-gamma release assays (IGARs) testing during biologic treatment course because of the moderate prevalence of tuberculosis in Taiwan. $\left[{ }^{1}\right.$ Although several studies have reported the risk of active tuberculosis infection in patients with rheumatoid arthritis (RA), the long-term serial IGARs testing in patients with RA is limited.

Objectives: The aims of this study is to use serial interferon-gamma release assays(IGRAs) as a screening tool in patients with rheumatoid arthritis treated with different biologic agents in Taiwan.

Methods: We retrospectively collected 189 patients with rheumatoid arthritis receiving biologic agents between 2013 to 2017 in Chang Gung Memorial hospita in Taiwan. All RA patients underwent semi-annual IGRA test during biologic agents treatment.

Results: The seroconversion rate of IGAR in patients with rheumatoid arthritis after biologic treatment is $6.9 \%$. The median seroconversion period is 19 months Out of the 13 patients with seroconversion, $8(61.5 \%)$ patients were treated with TNF block and $5(38.5 \%)$ patients were treated with non-TNF blocker. All the patients with seroconversion evaluate the possible active TB infection and $69.2 \%$ $(9 / 13)$ received chemoprophylactic therapy by isoniazid. There is no active tuberculosis infection during serial IGAR tests. The risk factor included high baseline TBAg-nil/nil (OR 3.44, Cl 6.64-1.78), leflunomide(OR 11.51, Cl 61.34-2.16).

Abstract THU0226 - Table 1

\begin{tabular}{|c|c|c|c|c|}
\hline & $\begin{array}{l}\text { Total } \\
(n=189)\end{array}$ & $\begin{array}{l}\text { Seroconversion } \\
(n=13)\end{array}$ & $\begin{array}{l}\begin{array}{l}\text { Non-seroconversion } \\
(\mathrm{n}=176)\end{array} \\
\end{array}$ & p-value \\
\hline Female & $162(14.3 \%)$ & $10(76.9 \%)$ & $152(86.4 \%)$ & 0.348 \\
\hline $\begin{array}{l}\text { Age (Meant } \\
\text { standard deviation) }\end{array}$ & $54.7 \pm 12.2$ & $60.0 \pm 13.6$ & $54.3 \pm 12.1$ & 0.104 \\
\hline $\begin{array}{l}\text { Disease duration } \\
\text { (Meant standard } \\
\text { deviation) }\end{array}$ & $4.9 \pm 1.4$ & $4.6 \pm 1.4$ & $4.9 \pm 1.4$ & 0.591 \\
\hline $\begin{array}{l}\text { Prednisolone Mean } \\
\text { dose (mg/day) }\end{array}$ & $\begin{array}{l}132 / 189(69.8 \%) \\
8.8 \pm 9.5\end{array}$ & $\begin{array}{l}9 / 13(69.2 \%) \\
8.3 \pm 3.5\end{array}$ & $\begin{array}{l}123 / 176(69.88 \%) \\
8.8 \pm 9.8\end{array}$ & 0.873 \\
\hline Methotrexate & $146(77.2 \%)$ & $9(69.2 \%)$ & $137(77.8 \%)$ & 0.475 \\
\hline Sulfasalazine & $99(52.4 \%)$ & $9(69.2 \%)$ & $90(51.1 \%)$ & 0.207 \\
\hline Leflunomide & $24(12.7 \%)$ & $5(38.5 \%)$ & $19(10.8 \%)$ & $0.015^{*}$ \\
\hline Hydroxychloroquine & $104(55 \%)$ & $11(84.6 \%)$ & $93(52.8 \%)$ & $0.026^{*}$ \\
\hline TNF blocker & $143(75.7 \%)$ & $8(61.5 \%)$ & $135(76.7 \%)$ & 0.219 \\
\hline Non-TNF blcoker & $46(24.3 \%)$ & $5(38.5 \%)$ & $41(23.3 \%)$ & 0.219 \\
\hline TB Ag-nil & $0.02 \pm 0.12$ & $0.14 \pm 0.12$ & $0.01 \pm 0.12$ & $<0.001^{*}$ \\
\hline Mitogen-nil & $6.66 \pm 5.2$ & $8.16 \pm 4.84$ & $6.52 \pm 5.23$ & 0.278 \\
\hline DAS28 & $6.06+1.09$ & $6.42 \pm 0.91$ & $6.03+1.1$ & 0.261 \\
\hline Characteristics & \multicolumn{2}{|c|}{$\mathrm{OR}(95 \% \mathrm{Cl})$} & pvalue & \\
\hline Age & \multicolumn{2}{|c|}{$1.02(1.09,0.95)$} & 0.474 & \\
\hline Disease duration & \multirow{2}{*}{\multicolumn{2}{|c|}{$1.31(2.36,0.72)$}} & 0.366 & \\
\hline TB Ag-nil/nil & \multirow{2}{*}{\multicolumn{2}{|c|}{$\begin{array}{l}3.44(6.64,1.78) \\
11.51(61.34,2.16)\end{array}$}} & $<0.001^{*}$ & \\
\hline Leflunomide & & & $0.004^{*}$ & \\
\hline Hydroxychloroquine & \multicolumn{2}{|c|}{$6.49(43.88,0.96)$} & 0.055 & \\
\hline
\end{tabular}

Conclusions: Our study revealed patients with rheumatoid arthritis receiving long-term biologic therapy had a low rate IGAR seroconversion rate (6.9\%) Patients with background Leflunomide had higher odds ratio of seroconversion. However, the clinical importance of IGAR serocoversion remain controversial and further long-term large scale investigation is needed.

\section{REFERENCE:}

[1] Chen $\mathrm{Y}-\mathrm{H} .2015$ TRA revised recommendations for screening and management of tuberculosis infection in patients before and after anti-tumor necrosis factor-alpha biological treatment. 2015;29:1-8.

Disclosure of Interest: None declared

DOI: 10.1136/annrheumdis-2018-eular.1456 